\title{
Composición química y degradación de cuatro especies de Pennisetum sp
}

\section{Chemical composition and degradation four species of Pennisetum sp}

\author{
${ }^{\bullet}$ Alexandra E. Barrera-Álvarez ${ }^{1,2}$, Juan H. Avellaneda-Cevallos ${ }^{1,2,3}$, Edwin O. Tapia-Moreno ${ }^{1}$, \\ Mayra M. Peña-Galeas ${ }^{4}$, Carlos A. Molina-Hidrovo ${ }^{3}$, Lola M. Casanova Ferrin ${ }^{3}$ \\ ${ }^{1}$ Facultad de Ciencias Pecuarias, Campus Finca Experimental "La María” km 7 vía Quevedo-El Empalme. \\ Universidad Técnica Estatal de Quevedo.EC.120501.Quevedo,Ecuador.•barreraalvarez@yahoo.com; etapia@uteq.edu.ec \\ ${ }^{2}$ Programa de Maestría en Producción Animal, Universidad Tecnológica Equinoccial Sede Santo Domingo, \\ Ecuador. javellaneda@ute.edu.ec \\ ${ }^{3}$ Programa de Ganadería, Estación Experimental Tropical Pichilingue, Instituto Nacional de Investigaciones \\ Agropecuarias-INIAP, Quevedo,Ecuador. carlos.molina@iniap.gob.ec; lola.casanova.ferrin@gmail.com \\ ${ }^{4}$ Centro de Investigación y Desarrollo, Universidad Técnica de Babahoyo, Extensión Quevedo, Ecuador. \\ mayra.pena.galeas@gmail.com
}

\section{Resumen}

$\mathrm{L}$ a capacidad de degradación de los pastos de corte en la Costa, constituye una alternativa a la demanda de gramíneas para la alimentación de rumiantes. Se seleccionaron cuatro especies de pasto Pennisetum (elefante, king grass morado, maralfalfa y clon Cuba CT-115) a tres edades de corte $(30,45$ y 60 días) y se evaluó la dinámica degradativa en $0,3,6,12,24,48$ y 72 horas de incubación utilizando tres toros Brahman fistulados de $450 \mathrm{~kg}$ promedio. Se utilizó la técnica de las bolsas de nylon y se empleó un diseño de bloques al azar con un arreglo factorial $4 \times 3$ (especies forrajeras $x$ edad de corte). Los datos fueron expuestos a un análisis de varianza con la prueba de Tukey $(\mathrm{p} \leq 0.05)$ y con el programa estadístico (SAS, 2003). Se constató que el avance de la edad estuvo asociado con la disminución de la proteína, sus mejores proporciones fueron a los 30 días con $12.89 \%$, seguido por $12.19,11.53$ y $9.77 \%$ para el maralfalfa, CT-115, king grass y elefante, respectivamente. La mayor tasa de degradación in situ fue $\mathrm{p}<0.001$ para el pasto maralfalfa a los 30 días de corte, obteniendo materia seca $(88.85 \%)$, materia orgánica $(89.53 \%)$ y biodisponibilidad de cenizas $(85.24 \%)$ a las 72 horas de incubación. La liberación de estos componentes se vio influenciada con la madurez del forraje, por tanto, su alta degradación garantiza una cantidad de energía fermentable, disponible para el proceso de síntesis microbiana ruminal y es una buena alternativa de uso para los ganaderos.

Palabras clave: elefante, king grass morado, maralfalfa, clon Cuba CT-115, edad de corte, degradación in situ.

Recibido: 30-abril-2015. Recibido en forma corregida: 29-mayo-2015.

Aceptado: 30-julio-2015.

Publicado como ARTÍCULO CIENTÍFICO en Ciencia y Tecnología 8(2): 13-27 Diciembre de 2015
$\mathrm{T}$ The capacity to degradation of pastures cut on the coast, is an alternative to the demand of grasses for feeding ruminants. four species of grass Pennisetum (elephant, purple, king grass, maralfalfa and clone Cuba CT-115) at three cutting ages (30, 45 and 60 days) were selected and the degradative dynamics in $0,3,6,12,24$, 48 and 72 hours of incubation was evaluated, using three fistulated Brahman bulls $450 \mathrm{~kg}$ average. The technique of nylon bags was used and a randomized block design was used with a $4 \times 3$ factorial arrangement (forage species $x$ age cutoff). Data were subject to an analysis of variance with Tukey test $(\mathrm{p} \leq 0.05)$ and statistical software (SAS, 2003). It was found that increasing age was associated with decreased protein, best proportions was 30 days with $12.89 \%$, followed by $12.19,11.53$ and $9.77 \%$ for maralfalfa, CT-115, king grass and elephant, respectively. The highest rate of degradation was $\mathrm{p} \leq 0.001$ in situ for 30 days maralfalfa cutting, obtaining dry matter $(88.85 \%)$, organic matter $(89.53 \%)$ and ash bioavailability $(85.24 \%)$ after 72 hours incubation. The release of these components was influenced by forage maturity, thus its high degradation ensuring an amount of fermentable energy available to the rumen microbial synthesis process and it is a good alternative of use for livestock.

Key words: elephant, grass kings, purple, maralfalfa, clone Cuba CT-115, age cutoff, in situ degradation. 


\section{Introducción}

$\mathrm{E}$ 1 inadecuado uso de los pastos y forrajes en la alimentación del ganado, especialmente con gramíneas tropicales, es la subutilización de los carbohidratos estructurales del material vegetal como fuente energética, lo que interrumpe el uso óptimo de los nutrientes presentes en la dieta, y compromete el rendimiento del ganado (Sánchez, 2007).

Para que los pastos sean aprovechados íntegramente, se requiere saber qué especies son óptimos y su composición nutricional a diferentes edades de corte. Por otro lado, obtener mayor información sobre la degradación de los forrajes en el rumen, a través de la técnica de la cinética de degradación ruminal in situ, utilizando bolsas de nylon, mediante procesos bioquímicos y microbiológicos que ocurren para degradar los nutrientes, permite caracterizar la digestibilidad y la cinética de degradación de recursos forrajeros (Ceballos et al., 2008).

Esto plantea la necesidad de un mayor conocimiento de la riqueza nutritiva de las especies forrajeras del trópico, con vista a disminuir la utilización de granos y cereales $u$ otros alimentos que el hombre pueda consumir para la alimentación de los rumiantes. En tal virtud se considera importante que se utilice forraje tierno, o cuando las paredes celulares poseen la mayor concentración de pectinas, y no cuando maduran porque predominar la celulosa y la hemicelulosa que le otorgan mayor resistencia, o concentraciones crecientes de lignina, que infiltra la pared celular y le da mayor rigidez y el color amarillento característico del forraje maduro (Relling and Mattioli, 2003).

Según Valenciaga et al. (2007) es necesario reconocer que hay otros factores que pueden influir en los valores de digestibilidad y contribuyen a la variabilidad de los resultados. Estos están ligados con los niveles de ingestión voluntaria de alimentos y con el tiempo de retención en el tracto digestivo y, en especial, en los compartimentos gástricos. Por lo tanto, el estudio del valor nutritivo de los alimentos es primordial para la nutrición animal, estimando no solamente los análisis químicos, sino también los efectos de los procesos de digestión, absorción y metabolismo animal. Flores et al. (2003), expresa que para cubrir las necesidades animales, el valor nutritivo de los forrajes disponibles debe ser conocido con la máxima exactitud y el valor energético se comprueba por ensayos de digestibilidad con animales.

La digestibilidad de energía en los pastos es un factor importante que afecta el rendimiento de los animales, en este sentido, esta investigación tiene como objetivo evaluar el efecto de tres edades de corte sobre la composición química y degradabilidad in situ de Pennisetum purpureum (elefante), Pennisetum spp. (king grass morado), Pennisetum hibridum (maralfalfa) y Pennisetum purpureum (Clon Cuba CT-115) a los 30, 45 y 60 días de corte.

\section{Materiales y métodos}

I a investigación se realizó en la Finca Experimental L“La María", perteneciente a la Universidad Técnica Estatal de Quevedo, ubicada a $1^{\circ} 6^{\prime}$ de latitud sur y $79^{\circ}$ 29' de longitud oeste, a una altura de $120 \mathrm{msnm}$, en el $\mathrm{km} 7.5$ de la vía que conduce a Mocache. La ejecución tuvo una duración de 125 días promedio.

Técnicas y procedimiento. Se empleó la técnica de la bolsa de nylon, in situ (McDonald et al., 1999), donde las muestras de los pastos Pennisetum fueron fermentados dentro del rumen del animal a través de la suspensión de bolsas de nylon, sometidas a la acción microbial para determinar la degradabilidad ruminal afectando la tasa de desaparición de la materia seca, orgánica y biodisponibilidad de cenizas. Los equipos utilizados fueron una báscula con capacidad de 500 $\mathrm{kg}$, una balanza con capacidad de un $\mathrm{kg}$, un equipo de análisis de proteína total, una estufa, una estufa de aire forzado, un molino Thomas Willey, una balanza analítica, tres bovinos canulados de $450 \mathrm{~kg}$ de PV, 2.50 $\mathrm{kg}$ de cada especies de Pennisetum, 252 bolsas de nylon ANKON, 300 bandas de goma, overol y botas, guantes quirúrgicos, un computador, cámara fotográfica, registro de control.

Se valoró la degradabilidad de cada muestra en diferentes edades fenológicas, se utilizó el diseño de bloques al azar en arreglo factorial (4x3); especie Pennisetum $\mathrm{x}$ edad de corte de los pastos. Las variables evaluadas fueron degradabilidad in situ de la materia seca (MS), materia orgánica (MO) y biodisponibilidad de cenizas de los pastos.

Para la diferencia entre las medias de las especies forrajeras de la edad de corte y tratamientos en general, se empleó la prueba de Tukey al 5\% de probabilidad. Para el procesamiento de los datos se usó el procedimiento de los modelos lineales generales (PROC GLM) con el programa estadístico SAS (2003).

Preparación de las muestras. Se instaló el experimento de campo utilizando el banco de germoplasma establecido con las especies nombradas, iniciando con un corte de igualación a la altura de $15 \mathrm{~cm}$ de cada especie para el corte a los 30 días, seguido por cortes de 45 y 60 días de edad.

Para el análisis proximal (humedad parcial, humedad total, cenizas, proteína cruda y fibra cruda) 
según los procedimientos de AOAC (1980) se consideró las muestras del material original seco. Cada muestra fue desecada en un horno de laboratorio a $65^{\circ} \mathrm{C}$ por $48 \mathrm{~h}$, y molidas en un molino Thomas Willey, con una criba de $2 \mathrm{~mm}$. Estos análisis se realizaron antes de la incubación. Después de la incubación, los residuos de la digestión, lavados y secos, se utilizaron las muestras para calcular materia seca (MS\%), materia orgánica (MO\%), y biodisponibilidad de cenizas, mediante la técnica de Ørskov et al. (1980).

Para los análisis de degradación ruminal, se usaron las bolsas de nylon marca Ankon (10x20 cm; tamaño del poro $45 \mu \mathrm{m}$ ), identificadas y colocadas a la estufa por $48 \mathrm{~h}$ a $65^{\circ} \mathrm{C}$; posteriormente fueron pesadas en balanza analítica y registrado su peso seco. Seguido, las bolsas fueron llenadas con $10 \mathrm{~g}$ de forraje previamente molido (muestra con un 10\% de humedad aproximada), a continuación, las bolsas de nylon fueron selladas usando bandas de goma. Cada muestra de forraje fue incubado en cada bovino por periodo de tiempo de: 0 , 3, 6, 12, 24, 48 y 72 horas. Después de la extracción del rumen, las bolsas de nylon fueron lavadas en agua corriente fría, hasta que el lavado las limpie y descolore. Las bolsas fueron desecadas primeramente al ambiente y luego ingresadas a un horno de aire-forzado por $48 \mathrm{~h}$ a $65^{\circ} \mathrm{C}$, para finalmente ser pesadas.

\section{Resultados y discusión}

\section{Composición química}

Se observó que la edad de corte influyó en la composición química de los pastos (Bernal, 1994; Gojon-Báez et al., 1998; Herrera-Torres et al., 2010), presentandose una ligera diferencia en los valores de MS y MO con relación a la edad de corte $(30,45$ y 60 días), que pudiera ser por disponibilidad de agua, época del año, fertilidad de los suelos, entre otras (Mari et al., 2004; Robinson, 2005). Los valores proteicos obtenidos en los pastos, indican una disminución en la proteína al aumentar la edad de los pastos, presentando sus mejores proporciones a los 30 días con $12.89 \%$, seguido por $12.19,11.53$ y $9.77 \%$ para el maralfalfa, CT-115, king grass y elefante, respectivamente. Esta tendencia es respaldada con las afirmaciones de Márquez et al. (2007); Chacón-Hernández y Vargas-Rodríguez (2009); Villalobos (2012) quienes obtuvieron el mismo comportamiento en diferentes experimentos.

En relación con otras investigaciones, Molina (2005) determinó que el contenido de PC para el pasto maralfalfa a los 35,45 y 60 días fueron de $12.46,10.80$ y $7.12 \%$, respectivamente, similares a las obtenidas en ésta investigación $(12.89,11.96$, y $8.46 \%$ en orden a los 30, 45 y 60 días de corte), mientras que Porras y
Castellanos (2006) reportaron valores más bajos para el mismo cultivar a los 30,45 y 60 días $(9.75 ; 8.69$ y $5.35 \%)$. Según Ramírez et al. (2008) atribuye esta disminución a una reducción de la actividad metabólica de la planta de manera que conforme se cosecha el forraje a una edad mayor, la síntesis de compuestos proteicos en la planta es menor, haciendo que los valores de PC bajen.

Inversamente ocurre con la fibra, que aumenta con la edad de corte, mostrando valores más bajos a los 30 días con $25.09 ; 25.21 ; 29.69$ y $29.80 \%$ para el pasto elefante, king grass, maralfalfa y CT-115, respectivamente. El comportamiento productivo es similar a los obtenidos por Verdecia et al. (2008) quienes determinaron el rendimiento y algunos componentes del valor nutritivo del Panicum maximum cv. Tanzania a diferentes edades de rebrote. Acerca del porcentaje de cenizas, los análisis muestran una leve diferencia entre los pastos estudiados, sin embargo, el contenido de cenizas es una guía del contenido de materia orgánica (Herrera et al., 2008) por tanto, a mayor contenido de $\mathrm{MO}$, menor contenido mineral.

\section{Degradabilidad ruminal in situ de la materia seca}

Efecto de las especies. La degradabilidad ruminal in situ de la MS mostró diferencias $(\mathrm{p}<.001)$ entre las especies en las siete horas de incubación (Cuadro 1), prevaleciendo el pasto maralfalfa con $81.65 \%$ a las $72 \mathrm{~h}$, seguido por el CT-115 (78.53\%), king grass $(76.53 \%)$ y elefante $(72.57 \%)$. Esto pudo deberse a la individualidad bioquímica de cada planta (Fortes et al., 2009; Correa, 2011). Estas medidas están asociadas a características morfo-fisiológicas de las plantas que pueden variar con la especie, la variedad, el clima, el suelo y otros, pero esencialmente con el estado de madurez (Santana et al., 2010).

Las especies Pennisetum, son muy similares morfológicamente y nutritivamente entre sí, pero con alguna característica que lo diferencia en rendimiento como el clon CT-115 que sobresale en nutrientes, demostrando que se asimila más en el rumen.

Efecto de la edad de corte. Las mediciones de degradabilidad ruminal in situ de MS de los pastos elefante, king grass, maralfalfa y CT-115, a los 30,45 y 60 días de corte, mostraron diferencias $(\mathrm{p}<0.001)$ a las $0,3,12,24,48$ y 72 horas de incubación ruminal, demostrando que a medida que aumenta la edad de corte, la degradabilidad disminuye (Cuadro 2), consiguiendo la misma conducta Correa (2006) y, Chacón-Hernández y Vargas-Rodríguez (2009).

Varios autores sintetizan que al aumentar la edad de rebrote se incrementa la síntesis de carbohidratos estructurales, disminuyendo las formas solubles, y 
Cuadro 1. Efecto de las variedades de pasto elefante, king grass, maralfalfa y CT115 en la degradabilidad ruminal in situ de MS

\begin{tabular}{crrrrc}
\hline Horas incub. & Elefante & king grass & Maralfalfa & CT-115 & Prob. \\
\hline 0 & $18.30 \mathrm{c}$ & $21.24 \mathrm{~b}$ & $24.42 \mathrm{a}$ & $20.92 \mathrm{~b}$ & $<.0001$ \\
3 & $24.95 \mathrm{~b}$ & $25.04 \mathrm{~b}$ & $30.56 \mathrm{a}$ & $26.16 \mathrm{~b}$ & $<.0001$ \\
6 & $29.56 \mathrm{~b}$ & $31.92 \mathrm{~b}$ & $36.51 \mathrm{a}$ & $35.11 \mathrm{a}$ & $<.0001$ \\
12 & $43.10 \mathrm{c}$ & $45.93 \mathrm{bc}$ & $51.57 \mathrm{a}$ & $46.99 \mathrm{~b}$ & $<.0001$ \\
24 & $55.37 \mathrm{~b}$ & $58.39 \mathrm{~b}$ & $65.92 \mathrm{a}$ & $62.59 \mathrm{a}$ & $<.0001$ \\
48 & $67.40 \mathrm{c}$ & $71.88 \mathrm{~b}$ & $76.37 \mathrm{a}$ & $74.15 \mathrm{ab}$ & $<.0001$ \\
72 & $72.57 \mathrm{c}$ & $76.53 \mathrm{~b}$ & $81.65 \mathrm{a}$ & $78.53 \mathrm{ab}$ & $<.0001$ \\
\hline
\end{tabular}

Medias seguidas por la misma letra en la misma fila, no difieren estadísticamente (Tukey, $\mathrm{p}>0.05$ ).

se afecta la calidad (Santana et al., 2010; GonzálezGarduño et al., 2011; Nava et al., 2013). Sin embargo, es conocido que cuando el forraje se hace más maduro se incrementan los contenidos de los nutrientes menos digeribles, aquellos constituyentes de la pared celular (celulosa, hemicelulosa, lignina), mientras que los más aprovechables por los animales decrecen en cuanto a su densidad (Bosch et al., 1992). Lara et al. (2010) concluyen que el pasto Dichanthium aristatum (Angleton), debe cosecharse hasta los 21 días para aprovechar al máximo el valor nutritivo de esta gramínea, por el contrario, si se desea conservar más cantidad de forraje, la mayor producción se logra a los 49 días con disminución en el aprovechamiento.

Efecto de la especie $x$ edad de corte. Los resultados de la degradabilidad in situ de la MS derivada de los pastos estudiados tuvieron una tendencia polinómica, obteniendo incrementos significativos en los siete tiempos de degradación ruminal $(\mathrm{p}<0.01)$. Las respuestas de digestibilidad de la MS entre la interacción especie por frecuencia de corte, varían con la variedad de pasto $(\mathrm{p}<.001)$. Los datos presentados en la figura 1 determinan que la mayor degradabilidad ocurrió para el pasto maralfalfa desde las cero horas hasta las 72 horas de incubación a los 30 días de edad siendo decrecientes a los 45 y 60 días (Cuadro 3). En estas estimaciones se considera que el corte a los 30 días se aprovecha al máximo el contenido nutricional del forraje, gracias a una digestibilidad mayor.

El comportamiento de la degradación de la MS de los pastos a los 30, 45 y 60 días de corte fue muy similar. Se observó la mayor extensión de degradación de la MS a los 30 días, en todos los tratamientos, para luego demostrar un incremento más lento, hasta las $72 \mathrm{~h}$ (Figura 1), lo cual indica que los nutrientes de los forrajes son utilizados de forma inmediata por la microbiota ruminal (Gojon-Báez et al., 1998; Herrera-

Cuadro 2. Efecto de las edades de corte en la degradabilidad ruminal in situ de MS

\begin{tabular}{ccccr}
\hline Horas incub. & 30 días & 45 días & 60 días & Prob. \\
\hline 0 & $24.21 \mathrm{a}$ & $20.09 \mathrm{~b}$ & $19.36 \mathrm{~b}$ & $<.0001$ \\
3 & $29.03 \mathrm{a}$ & $27.39 \mathrm{a}$ & $23.62 \mathrm{~b}$ & $<.0001$ \\
6 & $34.22 \mathrm{a}$ & $33.99 \mathrm{ab}$ & $31.61 \mathrm{~b}$ & 0.025 \\
12 & $49.29 \mathrm{a}$ & $47.31 \mathrm{a}$ & $44.11 \mathrm{~b}$ & 0.000 \\
24 & $65.32 \mathrm{a}$ & $60.19 \mathrm{~b}$ & $56.19 \mathrm{c}$ & $<.0001$ \\
48 & $77.19 \mathrm{a}$ & $73.05 \mathrm{~b}$ & $67.11 \mathrm{c}$ & $<.0001$ \\
72 & $81.88 \mathrm{a}$ & $77.43 \mathrm{~b}$ & $72.65 \mathrm{c}$ & $<.0001$ \\
\hline
\end{tabular}

Medias seguidas por la misma letra en la misma fila, no difieren estadísticamente (Tukey, $\mathrm{p}>0.05$ ). 


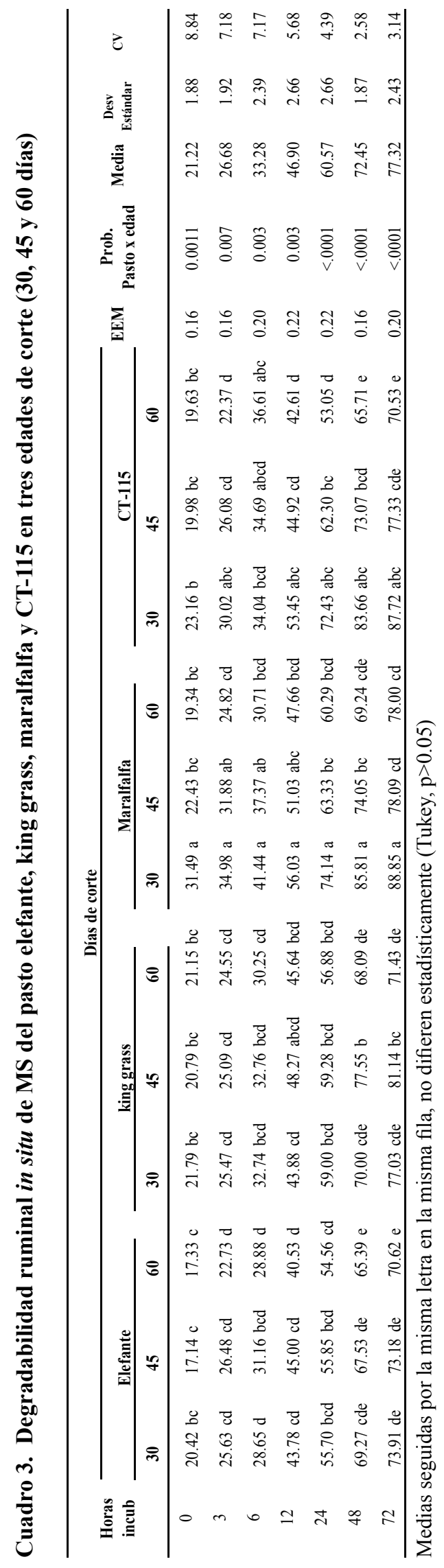



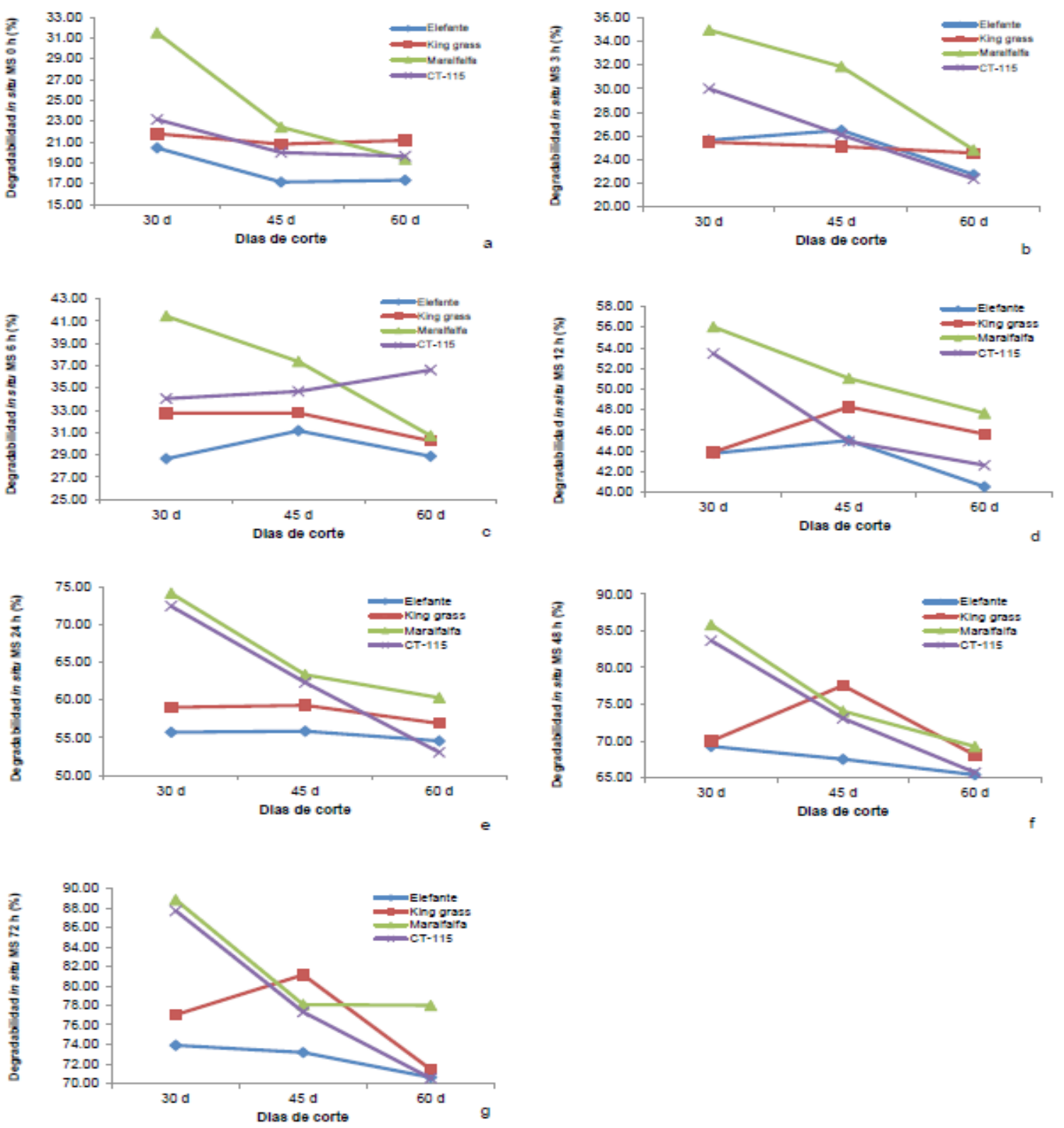

Figura 1. Interacción de la edad por variedad de Pennisetum. Degradabilidad in situ de la MS a las 0 h (a), 3 h (b), 6 h (c), 12 h (d), 24 h (e), 48 h (f) y 72 h (g) de incubación

Torres et al., 2010). Estos resultados están relacionados con los reportados por González et al. (2011) que sugieren realizar los períodos de corte para el pasto elefante, entre los 42 y 56 días, cuando el cultivo manifiesta el volumen y calidad de forraje conveniente para la alimentación de rumiantes. Esta tendencia de degradación concuerda con investigaciones realizadas por Chacón-Hernández y Vargas-Rodríguez (2009) en digestibilidad y calidad de king grass a los 60,75 y 90 días a seis tiempos $(2,6,12,24,48$ y 60 h) presentando valores de 79.24, 67.85 y $64.11 \%$ de degradabilidad, respectivamente, luego de las 48 horas y 60 días de crecimiento; expresando que la fase de mayor actividad de degradabilidad ruminal ocurre hasta las 60 horas (Rodríguez et al., 2011) y que la calidad del forraje disminuye conforme aumenta la edad de cosecha, recomendando la edad de corte hasta los 60 días de crecimiento. Similares resultados obtuvo Correa (2006), con el pasto maralfalfa (Pennisetum sp) cortados a los 56 y 105 días, e incubados durante 0,2 , 
$6,12,24,48$ y 72 horas en el rumen de cuatro vacas Holstein canuladas, utilizando una bolsa para cada tiempo en cada animal, se redujo la degradabilidad de la MS a medida que avanza la edad de rebrote, obteniendo $56.90 \%$ a los 56 días y $49.10 \%$ a los 105 días de corte. Esto lo atribuyó al alto potencial para la producción de biomasa (Correa et al., 2004) del pasto de corte, que extrae grandes cantidades de nutrientes exigiendo en la misma proporción labores de fertilización que certifiquen la continuidad del cultivo sin poner en riesgo la productividad del tierra. De hecho, Gómez et al. (2007) al evaluar la degradabilidad ruminal de los pastos pangola, swazi y estrella a los 21, 28 y 35 días de corte, a través de la técnica in situ, concluyen que edad adecuada es a los 21 días, porque tuvo el mejor aprovechamiento, fue más digestible por la velocidad con que atravesaron el tracto del animal absorbiéndose al máximo los nutrientes de dichos materiales.

\section{Degradabilidad ruminal in situ de la materia orgánica}

Efecto de las especies. El efecto de las variedades de pastos en la desaparición de la MO fue significativa $(\mathrm{p}<0.0001)$. Los resultados muestran una tendencia similar entre ellos (Cuadro 4); pero la curva durante todo el periodo de incubación fue mayor $(82.16 \%)$ para el maralfalfa, presentando mejor porcentaje de degradación ruminal a las 72 horas de incubación. Esta tendencia fue similar en un estudio realizado en degradabilidad de la MO del pasto Swazi en cinco tiempos de incubación $(12,24,36,48$ y 72 h) a tres edades de corte $(21,28$ y $35 \mathrm{~d})$ obteniendo resultados

Cuadro 4. Efecto de las variedades de pasto elefante, king grass, maralfalfa y CT-115 en la degradabilidad ruminal in situ de MO

\begin{tabular}{ccccccc}
\hline $\begin{array}{c}\text { Horas } \\
\text { incub. }\end{array}$ & Elefante & $\begin{array}{c}\text { king } \\
\text { grass }\end{array}$ & $\begin{array}{c}\text { Maral- } \\
\text { falfa }\end{array}$ & CT-115 & Prob. \\
\hline 0 & $12.58 \mathrm{~b}$ & $13.25 \mathrm{~b}$ & $17.87 \mathrm{a}$ & $14.75 \mathrm{ab}$ & 0.003 \\
\hline 3 & $20.46 \mathrm{~b}$ & $17.81 \mathrm{~b}$ & $24.98 \mathrm{a}$ & $20.43 \mathrm{~b}$ & $<.0001$ \\
\hline 6 & $25.04 \mathrm{~b}$ & $24.93 \mathrm{~b}$ & $31.38 \mathrm{a}$ & $30.30 \mathrm{a}$ & $<.0001$ \\
12 & $40.20 \mathrm{~b}$ & $41.66 \mathrm{~b}$ & $48.32 \mathrm{a}$ & $43.32 \mathrm{~b}$ & $<.0001$ \\
24 & $53.60 \mathrm{~b}$ & $56.33 \mathrm{~b}$ & $64.37 \mathrm{a}$ & $61.21 \mathrm{a}$ & $<.0001$ \\
48 & $67.40 \mathrm{c}$ & $71.92 \mathrm{~b}$ & $76.27 \mathrm{a}$ & $74.26 \mathrm{ab}$ & $<.0001$ \\
72 & $73.23 \mathrm{c}$ & $77.04 \mathrm{~b}$ & $82.16 \mathrm{a}$ & $79.09 \mathrm{ab}$ & $<.0001$ \\
\hline
\end{tabular}

Medias seguidas por la misma letra en la misma fila, no difieren estadísticamente (Tukey, p>0.05). de desaparición del material evaluado de 72.32, 70.14 y $70.92 \%$ a los 21 días y a las 72 horas de incubación (Gómez et al., 2007).

Por la naturaleza de cada uno de los pastos se observa que los porcentajes más altos de desaparición progresiva fueron hasta las 48 horas de incubación, manteniendo un comportamiento homogéneo en todas las especies.

Efecto de la edad de corte. La digestibilidad de la MO de las cuatro especies a tres edades de corte fueron significativos $(\mathrm{p}<0.001)$ a las $0,3,12,24,48$ y 72 horas de incubación, viéndose disminuidos con el aumento de la edad (Cuadro 5).

Los resultados presentaron la mayor extensión de degradación ruminal a los 30 días de corte con $84.40 \%$, seguido por 45 y 60 días (78.07 y $73.17 \%$, respectivamente). Similares apreciaciones tuvieron Ramírez et al. (2009), en el período poco lluvioso, el efecto de la edad; 30 (63.30\%), 45 (60.20\%), 60 $(56.50 \%)$ y $75(55.40 \%)$, evidenciándose mejores resultados a los 30 días de rebrote para la digestibilidad de la MO, valores inferiores comparado con este estudio, respaldando que esta consecuencia pudo estar influenciada por un aumento de las partes más leñosas de la planta, incremento de la síntesis de carbohidratos estructurales, como celulosa y hemicelulosa; así como por el efecto del bloqueo que ejerce la lignina sobre la acción enzimática de los microorganismos del retículo rumen (Fernández et al., 1991).

Por otra parte, Vergara-López y Araujo-Febres (2006) evaluaron la producción, composición química y degradabilidad ruminal in situ de Brachiaria humidicola en la época seca, obteniendo comportamientos variables de MO en las edades de

Cuadro 5. Efecto de la edad de corte en la degradabilidad ruminal in situ de MO

\begin{tabular}{ccrrr}
\hline $\begin{array}{c}\text { Horas } \\
\text { incub }\end{array}$ & 30 días & 45 días & 60 días & Prob. \\
\hline 0 & $17.94 \mathrm{a}$ & $13.33 \mathrm{~b}$ & $12.57 \mathrm{~b}$ & 0.000 \\
3 & $23.48 \mathrm{a}$ & $21.8 \mathrm{a}$ & $17.49 \mathrm{~b}$ & $<.0001$ \\
6 & $29.14 \mathrm{a}$ & $28.28 \mathrm{a}$ & $26.31 \mathrm{a}$ & 0.069 \\
12 & $46.01 \mathrm{a}$ & $43.86 \mathrm{a}$ & $40.59 \mathrm{~b}$ & 0.001 \\
24 & $63.77 \mathrm{a}$ & $58.42 \mathrm{~b}$ & $54.44 \mathrm{c}$ & $<.0001$ \\
48 & $77.37 \mathrm{a}$ & $73.14 \mathrm{~b}$ & $66.88 \mathrm{c}$ & $<.0001$ \\
72 & $82.4 \mathrm{a}$ & $78.07 \mathrm{~b}$ & $73.17 \mathrm{c}$ & $<.0001$ \\
\hline
\end{tabular}

Medias seguidas por la misma letra en la misma fila, no difieren estadísticamente (Tukey, p>0.05). 
corte; $14 \mathrm{~d}(89.64 \%), 28 \mathrm{~d}(88.56 \%), 42 \mathrm{~d}(88.69 \%)$, $56 \mathrm{~d}(89.68 \%), 70 \mathrm{~d}(88.88 \%), 84 \mathrm{~d}(89.22 \%)$ y 98 d (87.97\%). Herrera y Hernández (1989) explican que la acumulación de MO posiblemente se debió al incremento en la formación de carbohidratos estructurales, sin embargo, mantienen niveles más o menos constantes entre edades de corte.

Efecto de la especie $x$ edad de corte. Los factores especies y edad de corte en época seca, tuvieron efectos
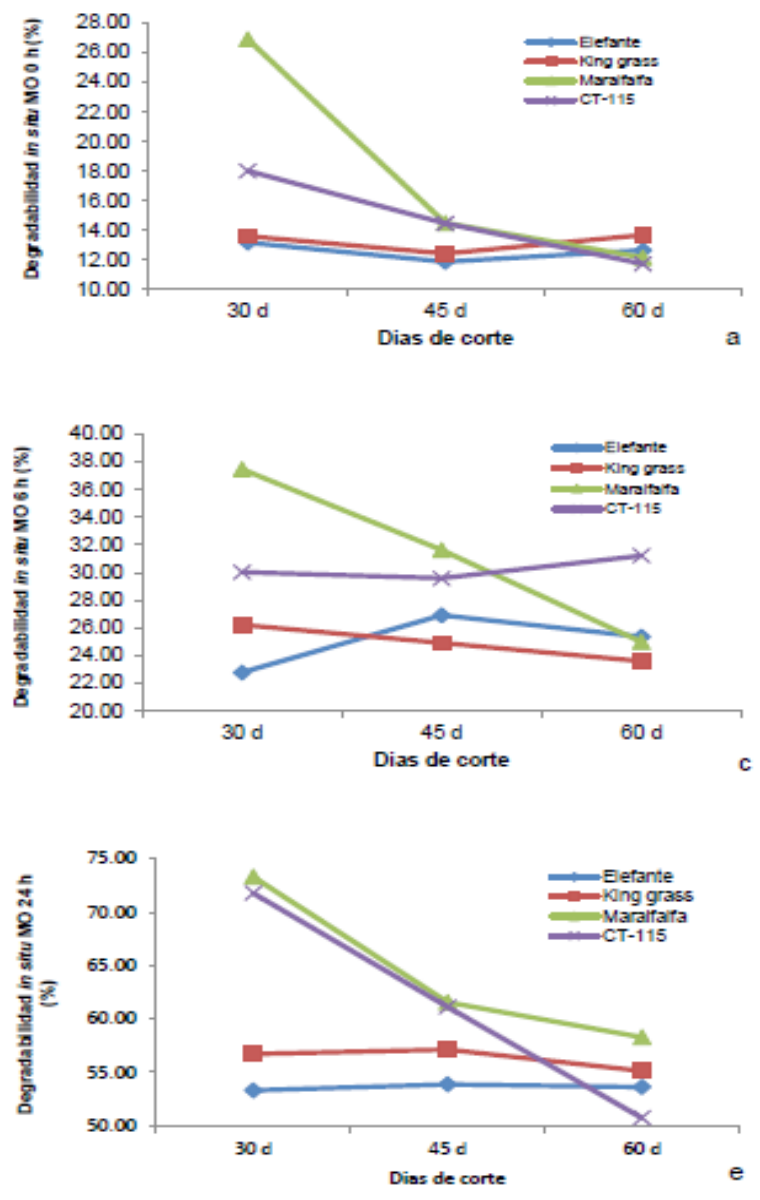
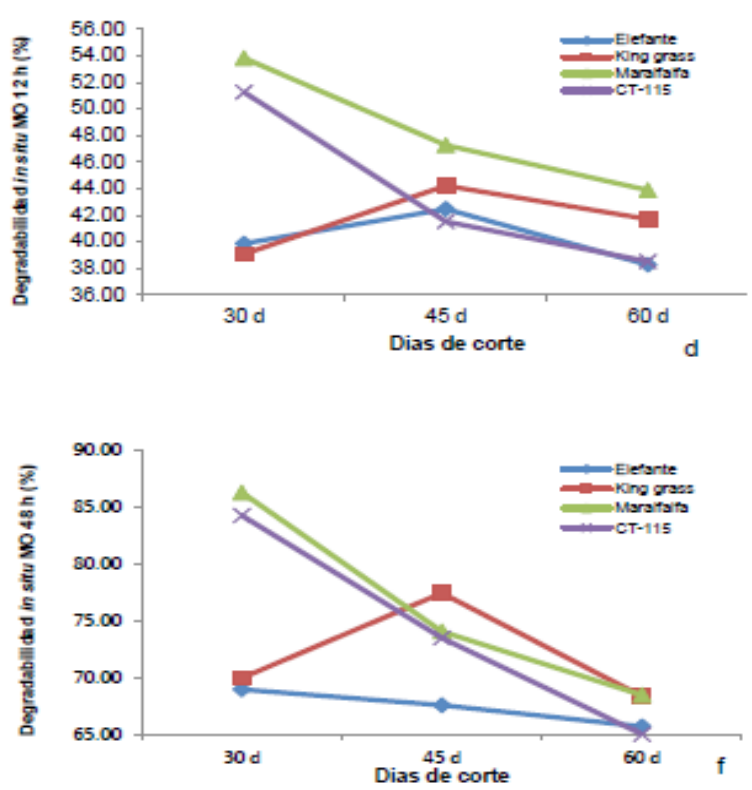

significativos en los contenidos de degradabilidad in situ de la MO en la planta, y los porcentajes de desaparición fueron superiores $(\mathrm{p}<.001)$ en la interacción de los dos factores en todas las horas de incubación (Figura 2).

De manera que, la degradabilidad de la materia orgánica de los sustratos presenta la mayor tasa de degradación para la especie maralfalfa con 89.53, 78.44 y $78.49 \%$ a los 30,45 y 60 días de corte, a las 72 horas de incubación (Cuadro 6); indicando la disponibilidad de nutrientes que esta especie posee para

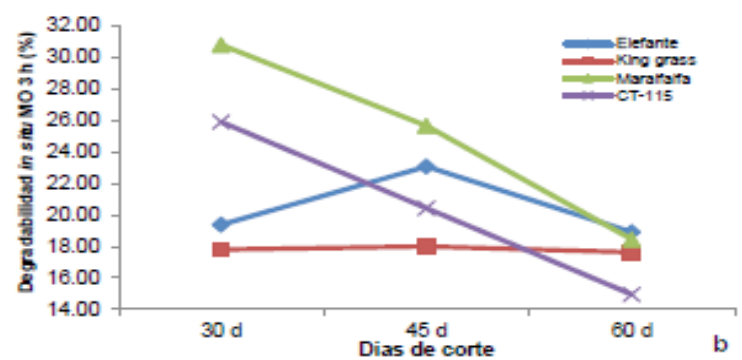

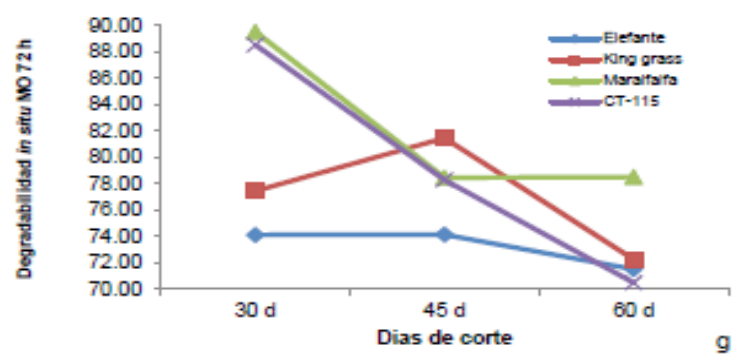

Figura 2. Interacción de la edad por variedad de Pennisetum. Degradabilidad in situ de la MO a las 0 h (a), 3 h (b), 6 h (c), 12 h (d), 24 h (e), 48 h (f) y 72 h (g) de incubación 


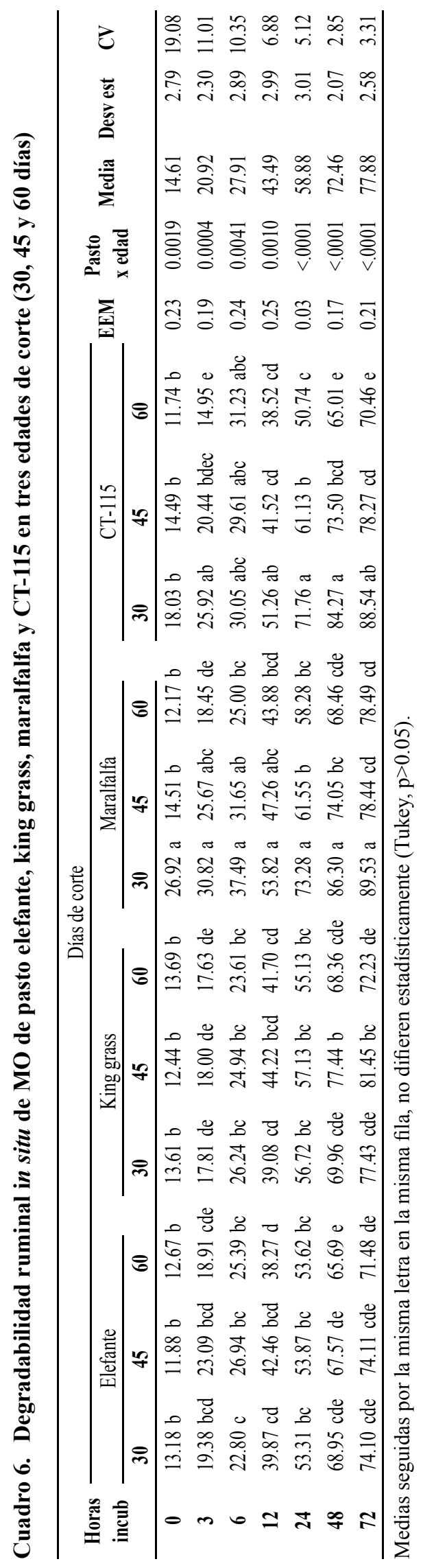


los microorganismos ruminales (Mould et al., 2005), además, es de señalar que el índice de degradablidad $(89.53 \%)$ permite afirmar que los pastos fueron cosechados en el punto óptimo de corte, puesto que los contenidos en los componentes de la pared celular no imposibilitaron que las bacterias y los demás microorganismos de la flora ruminal realicen una eficiente digestión de estos elementos (Martín et al., 2007).

\section{Biodisponibilidad de cenizas}

Efecto de las variedades. Las cenizas de los pastos están constituidas por el residuo inorgánico que queda después de que la materia orgánica se ha quemado (Soto et al., 2005; Cunuay y Choloquinga, 2011). Al evaluar el contenido de cenizas de las cuatro especies de Pennisetum presentan diferencias $(\mathrm{p}<.001)$ en las siete horas de incubación, sobresaliendo el maralfalfa a las $72 \mathrm{~h}$ con el $78.97 \%$ de absorción de minerales o biodisponibilidad de cenizas, seguido de CT-115, king grass y elefante (Cuadro 7). Al analizar el grado de desaparición mineral se aprecia que hasta las 24 horas existe un incremento de degradación para las variedades analizadas a excepción de maralfalfa y CT115 de 30 días que se extendieron hasta las $48 \mathrm{~h}$. El alto porcentaje de liberación de los minerales desaparece aprovechándose en el tracto gastrointestinal y está afectada por su ubicación y por su función dentro de las células vegetales (Caro y Correa, 2006).

En un estudio realizado por Delgado et al. (2005) sobre degradabilidad ruminal in situ de Pennisetum purpureum en búfalos, obtuvieron un porcentaje

Cuadro 7. Efecto de las variedades de pasto elefante, king grass, maralfalfa y CT-115 en la degradabilidad ruminal in situ de cenizas

\begin{tabular}{cccccc}
\hline $\begin{array}{c}\text { Horas } \\
\text { incub }\end{array}$ & Elefante & $\begin{array}{c}\text { king } \\
\text { grass }\end{array}$ & $\begin{array}{c}\text { Maral- } \\
\text { falfa }\end{array}$ & CT-115 & Prob. \\
\hline $\mathbf{0}$ & $49.49 \mathrm{~b}$ & $54.78 \mathrm{ab}$ & $58.58 \mathrm{a}$ & $51.96 \mathrm{~b}$ & 0.001 \\
$\mathbf{3}$ & $49.33 \mathrm{c}$ & $55.32 \mathrm{~b}$ & $59.72 \mathrm{a}$ & $54.77 \mathrm{~b}$ & $<.0001$ \\
$\mathbf{6}$ & $54.14 \mathrm{~b}$ & $61.27 \mathrm{a}$ & $63.29 \mathrm{a}$ & $59.35 \mathrm{a}$ & 0.000 \\
$\mathbf{1 2}$ & $58.84 \mathrm{c}$ & $63.78 \mathrm{~b}$ & $68.57 \mathrm{a}$ & $63.11 \mathrm{~b}$ & $<.0001$ \\
$\mathbf{2 4}$ & $64.84 \mathrm{c}$ & $67.02 \mathrm{bc}$ & $74.04 \mathrm{a}$ & $69.45 \mathrm{~b}$ & $<.0001$ \\
$\mathbf{4 8}$ & $67.21 \mathrm{c}$ & $71.72 \mathrm{~b}$ & $76.93 \mathrm{a}$ & $73.45 \mathrm{~b}$ & $<.0001$ \\
$\mathbf{7 2}$ & $68.72 \mathrm{c}$ & $74.41 \mathrm{~b}$ & $78.97 \mathrm{a}$ & $75.61 \mathrm{~b}$ & $<.0001$ \\
\hline
\end{tabular}

Medias seguidas por la misma letra en la misma fila, no difieren estadísticamente (Tukey, $\mathbf{p}>\mathbf{0 . 0 5}$ ). notable de nutrientes de rápido aprovechamiento por los microorganismos ruminales, estos valores altos de degradabilidad están relacionados con el potencial de los rumiantes para sostener niveles apropiados de producción, y es un indicativo potencial del alimento para proveer nutrientes a la flora ruminal (Cecconello et al., 2003).

Efecto de la edad de corte. Para el caso de la degradabilidad de la ceniza en los pastos elefante, king grass, maralfalfa y CT-115 fueron similares hasta las seis horas de incubación, mostrando diferencias $(\mathrm{p}<0.01)$ a partir de las 12 a 72 horas, notando la mayor desaparición a los 30 días de corte (Cuadro 8). La degradación de las cenizas durante el proceso fermentativo, indica que a mayor degradación del sustrato mayor biodisponibilidad de cenizas existe, sin embargo, no precisamente significa una mayor eficiencia por parte de los microorganismos en la utilización del sustrato. Según Patiño et al. (2011) expresan que los minerales no son transformados y que en el organismo se dan algunos procesos de reciclaje, siempre con pérdidas a través de la transpiración, leche, excretas, en animales gestantes son aprovechados por los tejidos fetales y los demás productos de la gestación. Sin embargo, carencias en minerales tienen un efecto negativo en el desempeño animal, en este sentido, el maralfalfa es una excelente opción de biodisponibilidad de cenizas (Figura 3).

Efecto de la especie $\mathbf{x}$ edad de corte. En todas las especies de mamíferos, los minerales son nutrientes esenciales para la vida. La biodisponibilidad mineral de los diferentes sustratos usados en la alimentación

Cuadro 8. Efecto de la edad de corte en la degradabilidad ruminal in situ de cenizas

\begin{tabular}{ccccc}
\hline $\begin{array}{c}\text { Horas } \\
\text { incub }\end{array}$ & 30 días & 45 días & 60 días & Prob. \\
\hline $\mathbf{0}$ & $54.24 \mathrm{a}$ & $52.79 \mathrm{a}$ & $54.08 \mathrm{a}$ & 0.644 \\
$\mathbf{3}$ & $55.39 \mathrm{a}$ & $54.21 \mathrm{a}$ & $54.76 \mathrm{a}$ & 0.119 \\
$\mathbf{6}$ & $58.53 \mathrm{a}$ & $61.45 \mathrm{a}$ & $58.57 \mathrm{a}$ & 0.099 \\
$\mathbf{1 2}$ & $64.74 \mathrm{a}$ & $63.95 \mathrm{ab}$ & $62.04 \mathrm{~b}$ & 0.010 \\
$\mathbf{2 4}$ & $72.55 \mathrm{a}$ & $68.87 \mathrm{~b}$ & $65.10 \mathrm{c}$ & $<.0001$ \\
$\mathbf{4 8}$ & $76.13 \mathrm{a}$ & $72.62 \mathrm{~b}$ & $68.24 \mathrm{c}$ & $<.0001$ \\
$\mathbf{7 2}$ & $79.21 \mathrm{a}$ & $74.24 \mathrm{~b}$ & $69.84 \mathrm{c}$ & $<.0001$ \\
\hline
\end{tabular}

Medias seguidas por la misma letra en la misma fila, no difieren estadísticamente (Tukey, $\mathbf{p}>\mathbf{0 . 0 5}$ ). 


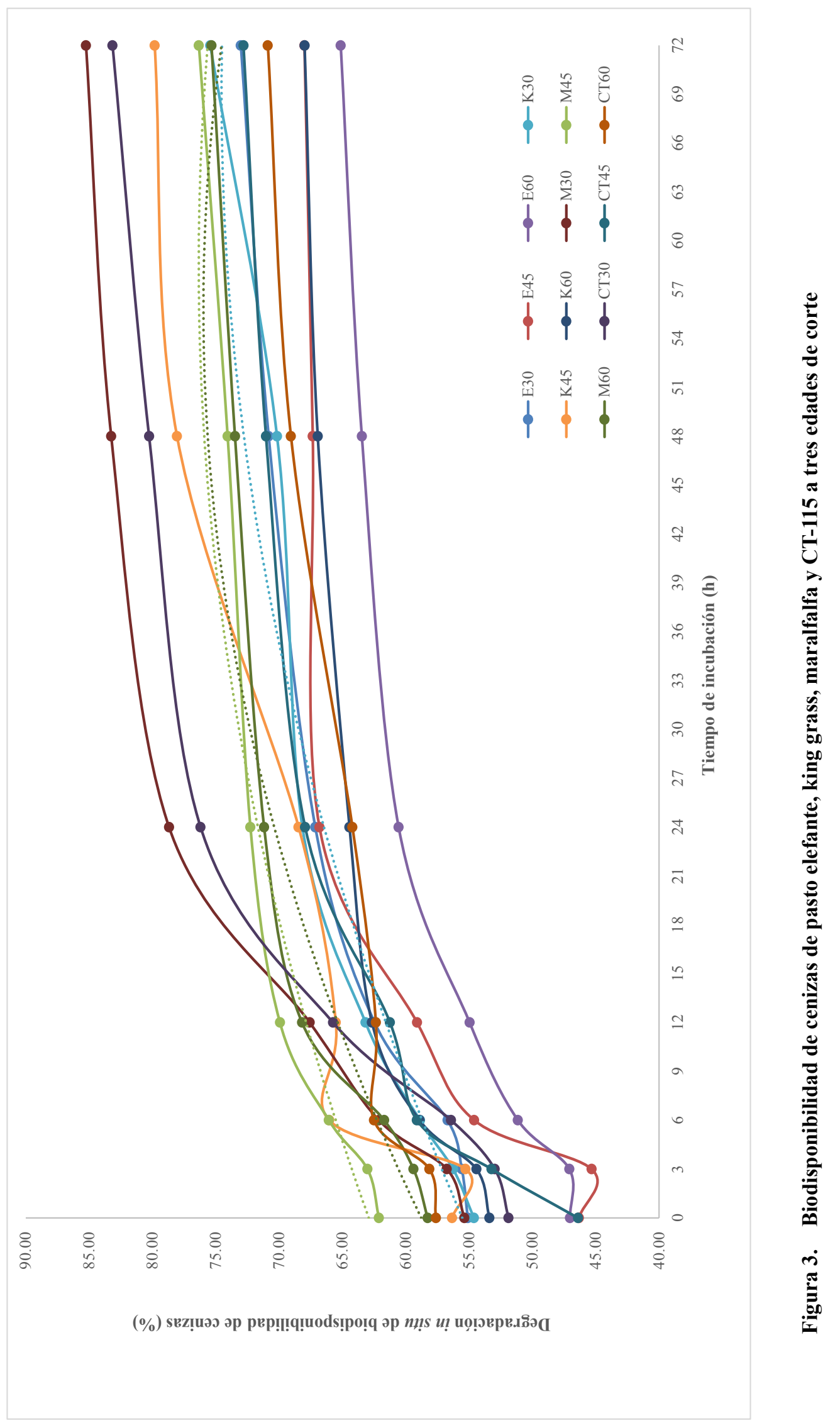


animal es variable, por lo tanto, debe ser analizada en los diferentes estados fisiológicos. Así, los datos de degradación de cenizas en las especies Pennisetum a 30,45 y 60 días de corte, fue diferente $(\mathrm{p}<0.001)$ a las $3,12,24,48$ y $72 \mathrm{~h}$, sin efecto significante a las $6 \mathrm{~h}$ de incubación (Cuadro 9).

La mayor biodisponibilidad de cenizas tuvo el pasto maralfalfa a los 30 días de corte $(85.24 \%)$ de asimilación ruminal a las $72 \mathrm{~h}$. El efecto de las especies mostraron tendencia a disminuir al aumentar la edad de corte, excepto el pasto king grass que tuvo un comportamiento semejante en todas las horas de incubación (Figura 4). Las variaciones entre especies y horas de degradabilidad están influenciada con la madurez del forraje para la liberación mineral de las pasturas, es decir, hay una disminución en la concentración de minerales (McDowell et al., 1997). Por tanto, las especies de Pennisetum aportan en forma eficiente con un porcentaje alto de biodisponibilidad de minerales de fácil degradabilidad en el rumen
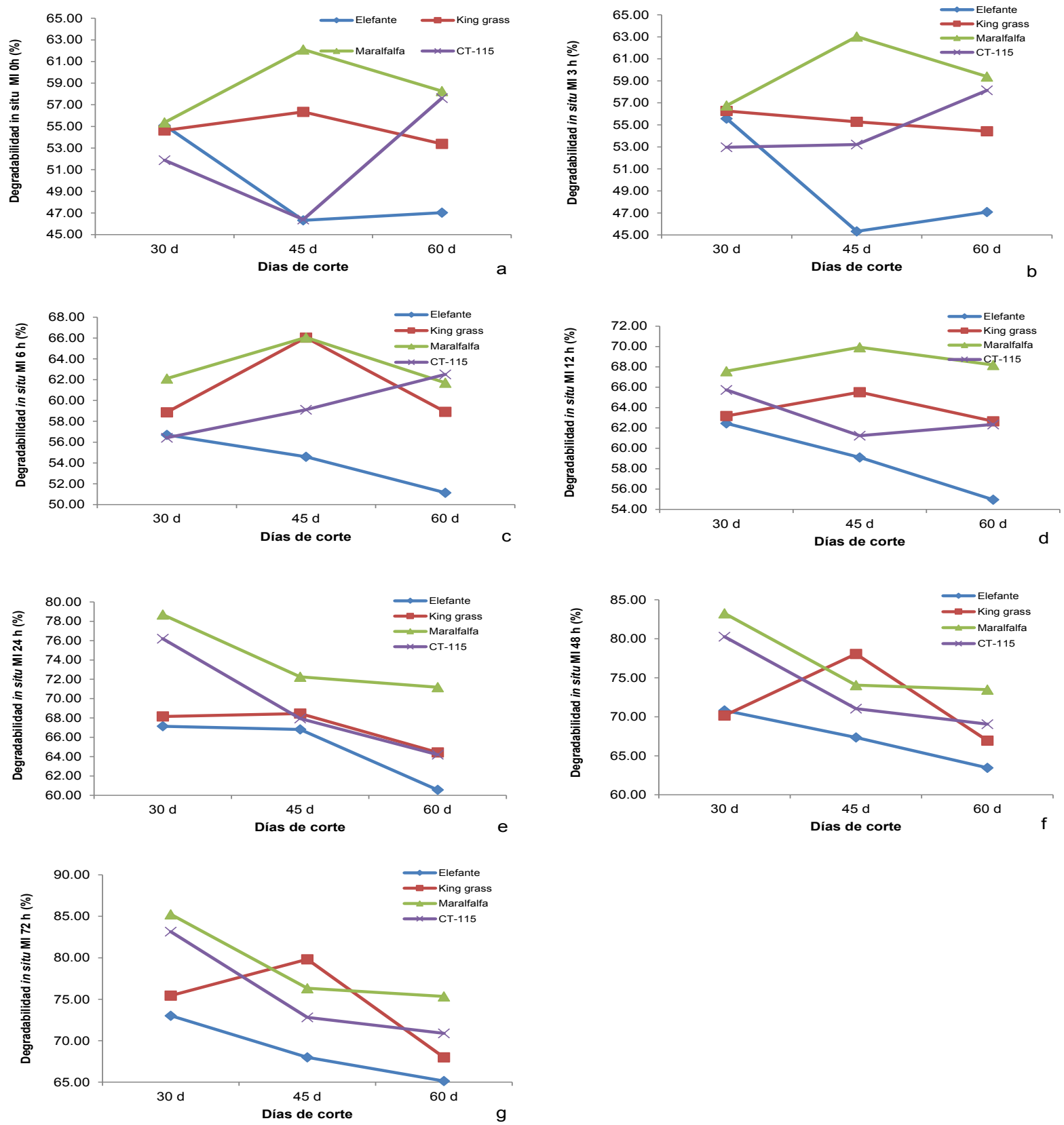

Figura 4. Interacción de la edad por variedad de Pennisetum. Degradabilidad in situ de la MI a las 0 h (a), 3 h (b), 6 h (c), 12 h (d), 24 h (e), 48 h (f) y 72 h (g) de incubación 
maximizando la función ruminal y logrando el máximo desempeño. Pues, se aconseja conocer la composición mineral de las pasturas, que componen la principal fuente de nutrientes, para realizar una atención correcta, real y económica de manejo.

\section{Conclusiones}

$\mathrm{E}^{1}$ pasto Pennisetum hibridum (maralfalfa) de 30 días de edad es una opción prometedora para la alimentación de los rumiantes y una buena alternativa de uso para los ganaderos, por su capacidad de suministrar altos valores de nutrientes degradables en el rumen. La degradación ruminal in situ de la Materia Seca, Materia Orgánica y Biodisponibilidad de Cenizas en los pastos Pennisetum es relativamente alta para las gramíneas del trópico en la época menos lluviosa. Su alta degradación garantiza una cantidad de energía fermentable, disponible para el proceso de síntesis microbiana ruminal.

\section{Agradecimiento}

A la Universidad Técnica Estatal de Quevedo por el financiamiento, y a la Universidad Autónoma del Estado de Hidalgo-México, por su participación como contraparte Internacional para el desarrollo del presente proyecto de investigación.

\section{Bibliografía}

AOAC. 1980. Official Methods of Analysis. Washington D.C.: Of the Association of Official Analytical Chemists.

Barahona, R., Sánchez, P. 2005. Limitaciones físicas y químicas de la digestibilidad de pastos tropicales y estrategias para aumentarla. Corpoica, 6(1): 6982.

Bernal, E. 1994. Pastos y forrajes tropicales. Producción y manejo. 3a ed. Bogotá D.C. (Col): Departamento de Publicaciones del Banco Ganadero.

Bosch, M., Tamminga, S., Post, G., Leffering, C., Muylaert, J. 1992. Influence of stage of maturity of grass silages on digestion processes in dairy cows. 1. Composition, nylon bag characteristics, digestibility and intake. Livestock Production Science, 32: 245-264.

Caro, F., Correa, H. 2006. Digestibilidad posruminal aparente de la materia seca, la proteína cruda y cuatro macrominerales en el pasto kikuyo (Pennisetum clandestinum) cosechado a dos edades de rebrote. Livestock Research for Rural Development, 18(10): 1-12.

Ceballos, A., Noguera, R., Bolívar, D., Posada, S. 2008. Comparación de las técnicas in situ de los sacos de nylon e in vitro (DaisyII) para estimar la cinética de degradación de alimentos para rumiantes. Livestock Research for Rural Development, 20(7): $1-11$.

Cecconello, G., Benezra, M., Ovispo, N. 2003. Composición química y degradabilidad ruminal de los frutos de algunas especies forrajeras leñosas de un bosque seco tropical. Zootecnia Tropical, 21, 149.

Chacón-Hernández, P., Vargas-Rodríguez, C. 2009. Digestibilidad y calidad del Pennisetum purpureum cv king grass a tres edades de rebrote. Universidad de Costa Rica. Costa Rica. Agronomía Mesoamericana, 20(2): 399-408.

Correa, C. 2006. Calidad nutricional del pasto maralfalfa (Pennisetum sp.) cosechado a dos edades de rebrote. Livestock Research Development, 18(6): $1-6$.

Correa, H. 2011. Limitaciones metabólicas para la producción bovina en el trópico bajo. En: Estrategias de alimentación en la ganadería y su impacto en la productividad. Revista CES Medicina Veterinaria y Zootecnia, 6(1): 9-19.

Correa, H., Ceron, J., Arroyave, H., Henao, Y., López, A. 2004. Pasto Maralfalfa: mitos y realidades. En: IV seminario internacional Competitividad en carne y leche. IV Seminario Internacional Competitividad en Carne y Leche (págs. 231-274). Medellín: Cooperativa Colanta.

Cunuay, J., Choloquinga, M. 2011. Evaluación de la adaptación del pasto maralfalfa (Pennisetum sp.), en dos pisos altitudinales con tres distancis de siembras en el campus Juan Lunardi y Naste del cantón Paute. Evaluación de la adaptación del pasto maralfalfa (Pennisetum sp.), en dos pisos altitudinales con tres distancias de siembras en el campus Juan Lunardi y Naste del cantón Paute. Cuenca, Azuay, Ecuador: Universidad Politécnica Salesina.

Delgado, D., Rosabal, Y., Cairo, J. 2005. Degradabilidad ruminal in situ de Pennisetum purpureum Cuba CT-115 en búfalos de río y Cebú comerciales. Instituto de Ciencia Animal Cuba. Revista Cubana de Ciencia Agrícola, 39(2): 187-192.

Fernández, R., de Chávez, M., Virgüez, D., García, M. 1991. Efecto de la frecuencia de corte sobre el rendimiento y valor nutritivo del pasto estrella (Cynodon nlemfuensis) en la unidad agroecológica 3e 144 del valle de Aroa. Zootecnia tropical, 9(2): 165-179. 
Flores, G., Castro, P., González-Arráez, A. 2003. Predicción de la digestibilidad "in vivo" de la materia orgánica de ensilajes de hierba y maíz por métodos de laboratorio. XIX Curso de especialización. Madrid: FEDNA.

Fortes, D., Herrera, R., González, S., García, M., Romero, A., Cruz, A. 2009. Comportamiento de los pigmentos fotosintéticos, según la edad de rebrote después del pastoreo de Pennisetum purpureum vc. Cuba CT-115 en la estación poco lluviosa. Revista Cubana de Ciencia Agricola 43(2): 183-186.

Gojon-Báez, D., Siqueiros-Beltrones, H., HernándezContreras, H. 1998. In situ ruminal digestibility and degradability of Macrocystis pyrifera and Sargassum spp. in bovine livestock. Ciencias Marinas 24(4): 463-481.

Gómez, H., Molina, V., Rubio, K. 2007. Evaluación de la degradabilidad ruminal de materiales forrajeros en dos sistemas de producción ganadera a través de la técnica in situ. Evaluación de la degradabilidad ruminal de materiales forrajeros en dos sistemas de producción ganadera a través de la técnica in situ. Buenos Aires, Buenos Aires, Argentina: Facultad de Ciencias Agronómicas, Universidad de El Salvador.

González, I., Betancourt, M., Fuenmayor, A., Lugo, M. 2011. Producción y composición química de forrajes de dos especies de pasto Elefante (Pennisetum sp.) en el Noroccidente de Venezuela. Zootecnia Tropical 29(1): 103-112.

Herrera, R., Fortes, D., García, M., Cruz, A., Romero, A. 2008. Estudio de la composición mineral en variedades de Pennisetum purpureum. Revista Cubana de Ciencia Agrícola 42(4): 395-401.

Herrera, R., Hernández, Y. 1989. Efecto de la edad de rebrote en algunos indicadores de la calidad de la bermuda cruzada-1. III. Porcentaje de hojas y rendimientos de materia seca y proteína bruta. Pastos y Forrajes (12): 77-81.

Herrera-Torres, E., Cerrillo-Soto, M., Juárez-Reyes, A., Murillo-Ortiz, M., Ríos-Rincón, F., ReyesEstrada, O., Bernal-Barragán, H. 2010. Efecto del tiempo de cosecha sobre el valor proteico y energético del forraje verde hidropónico de trigo. Interciencia 35(4): 284-289.

Lara, C., Oviedo, E., Betancur, A. 2010. Efecto de la época de corte sobre la composición química y degradabilidad ruminal del pasto Dichanthium Aristatum (Angleton). Zootecnia Tropical 28(2): 275-282.

Mari, L., Nussio, L., Schmidt, P. 2004. Magnitud de las alteraciones en la composición morfológica y el valor nutritivo de hierba Mandu mantenida a intervalos fijos entre cortes. Sociedad Brasileira de Zootecnia, 41.

Márquez, F., Sánchez, J., Urbano, D., Dávila, C. 2007. Evaluación de la frecuencia de corte y tipos de fertilización sobre tres genotipos de pasto elefante (Pennisetum purpureum). Rendimiento y contenido de proteína. Zootecnia Tropical 25(4): 253-259.

Martín, G., Noda, Y., Pentón, G., García, D., García, F., González, E., Ojeda, F., Milera, M., López, O., Ly, J., Leiva, L., Arece, J. 2007. La morera (Morus alba, Linn.): una especie de interés para la alimentación animal. Pastos y Forrajes 30(5): 1-7.

McDonald, P., Edwards, R.A., Greenhalgh, J.F.D., Morgan, C.A. 1999. Nutrición animal ( $5^{\mathrm{a}}$ ed.). Ed. Acribia, Zaragoza.

McDowell, L., Conrad, J., Ellis, G., Loosli, J. 1997. Minerals for grazing ruminants in tropical regions. Gainesville: University of Florida. Third Edition.

Molina, S. 2005. Evaluación agronómica y bromatológica del pasto Maralfalfa (Pennisetum purpureum) cultivado en el Valle del Sinú. Revista Facultad Nacional de Agronomía Colombia 58(1): 39.

Mould, F., Kliem, K., Morgan, R., Mauricio, R. 2005. In vitro microbial inoculum: A review of its function and properties. Animal Feed Science and Technology 123-124(1): 31-50.

Nava, J., Gutiérrez, E., Herrera, R., Zavala, F., Olivares, E., Treviño, J., Valdés, C. 2013. Rendimiento y composición química del pasto CT-115 (Pennisetum purpureum) establecido a dos densidades y en dos fechas de siembra en Marín, Nuevo León, México. Revista Cubana de Ciencia Agrícola 47(4): 419-424.

Ørskov, E., Hovell, F., Mould, F. 1980. The use of the nylon bag technique for the evaluation of feedstuffs. Tropical Animal Production 5: 195213.

Patiño, R., Da Silva, C., Pérez, J. 2011. Modelos de predicción de exigencias minerales para rumiantes. Revista Colombiana Ciencia Animal, 3(2): 344365.

Porras, D., Castellanos, L. 2006. Efecto de tres dosis de nitrógeno y tres edades de corte sobre el comportamiento de pasto Maralfalfa en zona bosque húmedo premontano. Memorias XIII Congreso Venezolano de Producción e Industria Animal (págs. 1-1). San Juan de los Morros, Guarico: AVPA.

Ramírez, J., Vega, M., Acosta, I., Verdecia, D. 2009. Caracterización nutritiva de las especies Brachiaria decumbens e hibrido en un suelo fluvisol de Cuba. Livestock Research for Rural 
Development 21(23): 1-12.

Ramírez, J., Verdecia, D., Leonard, I. 2008. Rendimiento y caracterización del Pennisetum Cuba CT169 en un suelo pluvisol. REDVET, 9(5): 1-10.

Relling, A., Mattioli, G. 2003. Fisiología digestiva y metabólica de los rumiantes. La Plata, Argentina: Facultad de Ciencias Veterinarias, EDULP.

Robinson, D. 2005. Fertilización y utilización de nutrientes en campos forrajeros de corte. Colombia: INTA, Bogotá.

Rodríguez, L., Torres, V., Martínez, R., Jay, O., Noda, A., Herrera, M. 2011. Models to estimate the growth dynamics of Pennisetum purpureum cv. Cuba CT-169. Cuban Journal of Agricultural Science 45(4): 349-354.

Sánchez, J. 2007. Utilización eficiente de las pasturas tropicales en la alimentación del ganado lechero. XI Seminario de pastos y forrajes en sistemas de producción animal. XI Seminario de pastos y forrajes en sistemas de producción animal (págs. 1-24). Barquisimeto, Venezuela: Universidad Centroccidental Lizandro Alvarado.

Santana, A., Pérez, A., Figueredo, M. 2010. Efectos del estado de madurez en el valor nutritivo y momento óptimo de corte del forraje napier (Pennisetum purpureum Schum.) en época lluviosa. Revista Mexicana de Ciencias Pecuarias 1(3): 277-286.
SAS. 2003. The SAS system for Windows. Release 9.1.3. Cary, North Carolina, USA: SAS Institute.

Soto, C., Valencia, A., Galvis, R., Correa, H. 2005. Efecto de la edad de corte y del nivel de fertilización nitrogenada sobre el valor energético y proteico del pasto kikuyo (Pennisetum clandestinum). Revista Colombiana Ciencias Pecuarias 18(1): 17-26.

Valenciaga, D., López, J., Chongo, B., Saliba, E., O.O. L., Cairo, J. 2007. Consumo y digestibilidad aparente de nutrientes en búfalas de río (Bubalus bubalis) alimentadas con Pennisetum purpureum vs. Cuba CT-115. Revista Cubana de Ciencia Agrícola 41(3): 249-252.

Verdecia, D., Ramírez, J., Leonard, I., López, P. 2008. Rendimiento y componentes del valor nutritivo del Panicum maximum cv. Tanzania. REDVET (Revista Electrónica de Veterinaria) 9(5): 1-9.

Vergara-López, J., Araujo-Febres, O. 2006. Producción, composición química y degradabilidad ruminal in situ de Brachiaria humidicola (RENDLE) Schweick en el bosque seco Tropical. Revista Científica (Maracaibo) 16(3): 239-248.

Villalobos, L. 2012. Fenología, producción y valor nutritivo del pasto alpiste (Phalaris arundinacea) en la zona alta lechera de Costa Rica. Agronomía Costarricense 36(1): 25-37. 